Quim. Nova, Vol. 31, No. 1, 94-97, 2008

\title{
ESTUdO COMPARATIVO DE MÉTODOS PARA A DETERMINAÇÃo DA CONCENTRAÇÃo DE CARBONO EM SOLOS COM ALTOS TEORES DE Fe (LATOSSOLOS)
}

\author{
Aline Segnini e Larissa Macedo dos Santos \\ Instituto de Química de São Carlos, Universidade de São Paulo, CP 780, 13560-970 São Carlos - SP/ Embrapa Instrumentação \\ Agropecuária, Empresa Brasileira de Pesquisa Agropecuária, CP 741, 13560-970 São Carlos - SP, Brasil \\ Wilson Tadeu Lopes da Silva* e Ladislau Martin-Neto \\ Embrapa Instrumentação Agropecuária, Empresa Brasileira de Pesquisa Agropecuária, CP 741, 13560-970 \\ São Carlos - SP, Brasil \\ Carlos Eduardo Borato \\ Interunidades em Ciência e Engenharia de Materiais, Universidade de São Paulo, CP 369, 13560-250 São Carlos - SP / Embrapa \\ Instrumentação Agropecuária, Empresa Brasileira de Pesquisa Agropecuária, CP 741, 13560-970 São Carlos - SP, Brasil \\ Wanderley José de Melo \\ Faculdade de Ciências Agrárias e Veterinárias, Universidade Estadual Paulista, 14884-900 Jaboticabal - SP, Brasil \\ Denizart Bolonhezi \\ Agência Paulista de Tecnologia dos Agronegócios Centro Leste, CP 271, 14001-970 Ribeirão Preto - SP, Brasil \\ Recebido em 16/2/07; aceito 23/5/07; publicado na web 3/12/07

\begin{abstract}
COMPARATIVE STUDY OF CARBON QUANTIFICATION METHODS IN SOIL WITH HIGH Fe CONTENTS (OXISOLS). Soil organic matter (SOM) plays an important role in physical, chemical and biological properties of soil. Therefore, the amount of SOM is important for soil management for sustainable agriculture. The objective of this work was to evaluate the amount of SOM in oxisols by different methods and compare them, using principal component analysis, regarding their limitations. The methods used in this work were Walkley-Black, elemental analysis, total organic carbon (TOC) and thermogravimetry. According to our results, TOC and elemental analysis were the most satisfactory methods for carbon quantification, due to their better accuracy and reproducibility.
\end{abstract}

Keywords: soil organic matter; carbon quantification methods; statistical evaluation.

\section{INTRODUÇÃO}

A matéria orgânica do solo (MOS) é um componente importante na definição da qualidade dos sistemas agrícolas ${ }^{1}$, bem como a capacidade do solo seqüestrar carbono da atmosfera. O conhecimento dos seus teores é fundamental em diversas áreas da ciência do solo e vários métodos têm sido utilizados para tal, como gravimetria por incineração em mufla, termogravimetria, cromatografia, oxidação da matéria orgânica (MO) por via úmida, (destacando-se os métodos preconizados por Schollenberger, Walkley-Black, Walkley-Black modificado) e oxidação por via $\mathrm{seca}^{2}$. Porém, há uma carência de informações consistentes quanto à definição e recomendação do método mais adequado para a obtenção de resultados confiáveis e satisfatórios ${ }^{3}$, principalmente para uso em metodologias não convencionais como métodos espectroscópicos, os quais necessitam dos valores precisos de carbono para a determinação do grau de humificação ${ }^{4}$.

O método de Walkley-Black ${ }^{5,6}$ é ainda hoje o mais empregado em laborátorios de solos devido a simplicidade e baixo custo, porém apresenta problemas analíticos e ambientais, devido ao uso de crômio. A utilização de analisadores elementares automáticos trouxe avanços consideráveis, permitindo análises rápidas e confiáveis ${ }^{7}$, contudo, o custo de cada análise e a manuntenção dos equipamentos são elevados. A metodologia de quantificação de carbono orgânico total ("TOC - Total Organic Carbon") é uma análise rápida, altamente reprodutível e facilmente automatizada. Os métodos

*e-mail: wilson@cnpdia.embrapa.br gravimétricos ${ }^{2,8}$ foram praticamente abandonados devido à baixa velocidade analítica e dificuldade de automação nos laboratórios, entretanto tem sido proposto seu uso em laboratórios de rotina na substituição do método de Walkley-Black².

Em função das dificuldades encontradas na seleção da melhor metodologia para a quantificação de carbono e da MOS, este trabalho teve como objetivos avaliar o teor de MO em amostras de latossolos a partir de diferentes métodos e comparar os métodos utilizados, através da análise multivariada, com a finalidade de estimar suas limitações na determinação do carbono e da MOS.

\section{PARTE EXPERIMENTAL}

O trabalho foi realizado com 25 amostras de solos coletadas em horizontes superfíciais e subsuperficiais de latossolos representativos da região norte do Estado de São Paulo. Experimentos em campo foram instalados na Faculdade de Ciências Agrárias e Veterinárias da UNESP, em Jaboticabal, e na Estação Experimental do Instituto Agronômico de Campinas (IAC) - Pólo Regional do Centro-Leste (APTA), em Ribeirão Preto. O clima das regiões de Jaboticabal e de Ribeirão Preto é do tipo Cwa, segundo a classificação climática de Köeppen. As precipitações médias anuais são de 1360 e $1427 \mathrm{~mm}$, com temperaturas máximas de 21 e $25{ }^{\circ} \mathrm{C}$ e mínimas de 15 e $19{ }^{\circ} \mathrm{C}$, e altitudes locais de 575 e 646 m para Jaboticabal e Ribeirão Preto, respectivamente 9 .

O experimento de Jaboticabal foi conduzido sob sistema de cultivo mínimo em Latossolo Vermelho eutroférrico (LVef) e Latossolo Vermelho distrófico (LVd), classificados conforme o Sis- 
tema Brasileiro de Classificação de Solos ${ }^{10}$, com e sem adição de lodo de esgoto, sendo que amostras de solo foram coletadas nas profundidades $0-10,10-20,20-40$ e 40-60 cm. O experimento de Ribeirão Preto foi conduzido sob sistema de cultivo mínimo, plantio direto e plantio convencional, em LVef, sendo as amostras de solo coletadas nas profundidades 0-10, 10-20 e 20-30 cm. A Tabela 1 representa as 25 amostras utilizadas.

As amostras de solo foram secas ao ar, peneiradas a $0,5 \mathrm{~mm}$, trituradas e submetidas à determinação dos teores de carbono e MOS.

\section{Determinação do teor de MOS por oxidação via úmida (Walkley-Black)}

A quantificação da MOS foi realizada a partir do teor de carbono orgânico em $1 \mathrm{~g}$ de amostra de solo, utilizando-se o fator de van Bemmelen $(1,724)$, com base no pressuposto de que a MOS contém $58 \%$ de carbono orgânico. A fórmula empregada ${ }^{5}$ foi MOS (g $\left.\mathrm{kg}^{-1}\right)=$ carbono orgânico $\left(\mathrm{g} \mathrm{kg}^{-1}\right) * 1,724$.

As análises químicas para a determinação do teor de carbono orgânico pelo método de Walkley-Black foram realizadas em duplicata no Laboratório de Preparo de Amostras da Embrapa Pecuária Sudeste, em São Carlos-SP. Após a realização das análises, o resíduo de sulfocrômica foi tratado segundo o Protocolo de Tratamento de Resíduos do Laboratório de Tratamento de Resíduos Químicos da Embrapa Pecuária Sudeste.

\section{Determinação da composição elementar (CNHS)}

A análise química para avaliação da composição elementar (carbono, nitrogênio, hidrogênio e enxofre) das amostras de solo foi realizada em duplicata a partir de $1 \mathrm{mg}$ de amostra, por combustão a $1000{ }^{\circ} \mathrm{C}$.

As medidas foram realizadas no equipamento Carlo-Erba Instruments modelo EA 1110, pertencente ao Instituto de Química da USP de São Carlos-SP e no equipamento da Carlo-Erba modelo EA 1108 pertencente à UFSCar.

\section{Determinação do TOC por via seca}

A determinação do TOC foi realizada em duplicata em $100 \mathrm{mg}$ de amostra de solo por oxidação a $900{ }^{\circ} \mathrm{C}$. Para os cálculos, fez-se uso de uma curva de calibração obtida com padrão de biftalato de potássio.

As medidas foram realizadas no aparelho Total Organic Carbon Analyser, TOC-V, da marca Shimadzu, acoplado ao Solid Sample Module, modelo SSM-5000 A, da marca Shimadzu, pertencente ao Laboratório de Química Ambiental, do Instituto de Química da USP de São Carlos-SP.

\section{Determinação da MOS por termogravimetria}

A análise termogravimétrica (TGA) foi realizada em duplicata em $10 \mathrm{mg}$ de amostra de solo, com taxa de aquecimento de $15^{\circ} \mathrm{C}$ $\min ^{-1}$ no intervalo de 25 a $700{ }^{\circ} \mathrm{C}$ sob atmosfera de ar. Foi utilizado um módulo termogravimétrico TGA Q-500, pertencente à Embrapa Instrumentação Agropecuária. $\mathrm{O}$ carbono $(\% \mathrm{~m} / \mathrm{m})$ foi estimado através da equação $\mathrm{Y}=3,72 \mathrm{x}+0,29$, onde $\mathrm{x}$ corresponde ao carbono orgânico (base seca) e Y à perda de massa de 100 a $300^{\circ} \mathrm{C}{ }^{2}$.

\section{Análise multivariada}

A análise de compentes principais (PCA) ${ }^{11}$ foi aplicada aos valores médios das 25 amostras. Foram tratadas duas matrizes $25 \times 3$ e $8 \times 4$. Os cálculos foram feitos no "software" "Matlab 6.1".

\section{RESULTADOS E DISCUSSÃO}

Observa-se que os valores de carbono (Tabela 2) obtidos pela aplicação do método de Walkley-Black são menores em relação

Tabela 1. Dados referentes às amostras de solo analisadas

\begin{tabular}{|c|c|c|c|c|}
\hline Amostras & Solos & Sistemas de manejo & Tratamentos & Profundidade $(\mathrm{cm})$ \\
\hline 1 & LVef & Plantio Direto & Sem adição de lodo & $0-10$ \\
\hline 2 & LVef & Plantio Direto & Sem adição de lodo & $10-20$ \\
\hline 3 & LVef & Plantio Direto & Sem adição de lodo & $20-30$ \\
\hline 4 & LVef & Cultivo Mínimo & Sem adição de lodo & $0-10$ \\
\hline 5 & LVef & Cultivo Mínimo & Sem adição de lodo & $10-20$ \\
\hline 6 & LVef & Cultivo Mínimo & Sem adição de lodo & $20-30$ \\
\hline 7 & LVef & Plantio Convencional & Sem adição de lodo & $0-10$ \\
\hline 8 & LVef & Plantio Convencional & Sem adição de lodo & $10-20$ \\
\hline 9 & LVef & Plantio Convencional & Sem adição de lodo & $20-30$ \\
\hline 10 & LVef & Cultivo Mínimo & Sem adição de lodo & $0-10$ \\
\hline 11 & LVef & Cultivo Mínimo & Sem adição de lodo & $10-20$ \\
\hline 12 & LVef & Cultivo Mínimo & Sem adição de lodo & $20-40$ \\
\hline 13 & LVef & Cultivo Mínimo & Sem adição de lodo & $40-60$ \\
\hline 14 & LVef & Cultivo Mínimo & Com adição de lodo & $0-10$ \\
\hline 15 & LVef & Cultivo Mínimo & Com adição de lodo & $10-20$ \\
\hline 16 & LVef & Cultivo Mínimo & Com adição de lodo & $20-40$ \\
\hline 17 & LVef & Cultivo Mínimo & Com adição de lodo & $40-60$ \\
\hline 18 & $\mathrm{LVd}$ & Cultivo Mínimo & Sem adição de lodo & $0-10$ \\
\hline 19 & LVd & Cultivo Mínimo & Sem adição de lodo & $10-20$ \\
\hline 20 & LVd & Cultivo Mínimo & Sem adição de lodo & $20-40$ \\
\hline 21 & LVd & Cultivo Mínimo & Sem adição de lodo & $40-60$ \\
\hline 22 & $\mathrm{LVd}$ & Cultivo Mínimo & Com adição de lodo & $0-10$ \\
\hline 23 & LVd & Cultivo Mínimo & Com adição de lodo & $10-20$ \\
\hline 24 & LVd & Cultivo Mínimo & Com adição de lodo & $20-40$ \\
\hline 25 & $\mathrm{LVd}$ & Cultivo Mínimo & Com adição de lodo & $40-60$ \\
\hline
\end{tabular}


aos obtidos por análise elementar e TOC, deixando claro que o método de Walkley-Black não é capaz de oxidar as formas de carbono do solo que se encontram mais protegidas ou complexadas com a fração mineral do solo, ocorrendo apenas uma oxidação parcial da MO, excluindo carvão e carbonatos ${ }^{12}$.

Tabela 2. Resultados de carbono obtidos pelos métodos de WalkleyBlack, análise elementar, carbono orgânico total (TOC) e análise termogravimétrica (TGA)

\begin{tabular}{|c|c|c|c|c|}
\hline Amostras & $\begin{array}{c}\text { Walkley- } \\
\text { Black } \\
\%(\mathrm{~m} / \mathrm{m})\end{array}$ & $\begin{array}{l}\text { Análise } \\
\text { Elementar } \\
\%(\mathrm{~m} / \mathrm{m})\end{array}$ & $\begin{array}{c}\text { TOC } \\
\%(\mathrm{~m} / \mathrm{m})\end{array}$ & $\begin{array}{c}\text { TGA } \\
\%(\mathrm{~m} / \mathrm{m})\end{array}$ \\
\hline 1 & $1,7 \pm 0,3$ & $3,1 \pm 0,5$ & $2,5 \pm 0,3$ & $2,46 \pm 0,08$ \\
\hline 2 & $1,7 \pm 0,2$ & $2,4 \pm 0,2$ & $2,2 \pm 0,3$ & $2,39 \pm 0,02$ \\
\hline 3 & $1,0 \pm 0,1$ & $2,3 \pm 0,5$ & $1,9 \pm 0,4$ & nd \\
\hline 4 & $1,3 \pm 0,4$ & $2,2 \pm 0,3$ & $2,5 \pm 0,1$ & $2,47 \pm 0,01$ \\
\hline 5 & $1,3 \pm 0,2$ & $2,0 \pm 0,2$ & $2,4 \pm 0,0$ & nd \\
\hline 6 & $0,9 \pm 0,2$ & $1,6 \pm 0,3$ & $1,8 \pm 0,4$ & nd \\
\hline 7 & $1,4 \pm 0,1$ & $2,3 \pm 0,1$ & $2,6 \pm 0,1$ & $2,52 \pm 0,07$ \\
\hline 8 & $1,5 \pm 0,3$ & $2,1 \pm 0,1$ & $2,4 \pm 0,3$ & nd \\
\hline 9 & $1,3 \pm 0,2$ & $1,9 \pm 0,2$ & $2,3 \pm 0,1$ & nd \\
\hline 10 & $1,9 \pm 0,4$ & $2,2 \pm 0,1$ & $1,9 \pm 0,3$ & nd \\
\hline 11 & $1,8 \pm 0,3$ & $1,8 \pm 0,1$ & $1,9 \pm 0,1$ & nd \\
\hline 12 & $1,3 \pm 0,3$ & $1,4 \pm 0,2$ & $1,4 \pm 0,1$ & nd \\
\hline 13 & $0,9 \pm 0,1$ & $1,5 \pm 0,8$ & $1,0 \pm 0,1$ & nd \\
\hline 14 & $2,0 \pm 0,3$ & $1,8 \pm 0,2$ & $2,0 \pm 0,2$ & nd \\
\hline 15 & $1,8 \pm 0,4$ & $1,6 \pm 0,3$ & $1,9 \pm 0,2$ & nd \\
\hline 16 & $1,6 \pm 0,2$ & $1,5 \pm 0,9$ & $1,7 \pm 0,2$ & $2,16 \pm 0,02$ \\
\hline 17 & $1,0 \pm 0,1$ & $1,0 \pm 0,1$ & $1,2 \pm 0,1$ & $2,34 \pm 0,08$ \\
\hline 18 & $1,3 \pm 0,2$ & $0,9 \pm 0,4$ & $1,2 \pm 0,1$ & $0,82 \pm 0,10$ \\
\hline 19 & $1,1 \pm 0,2$ & $1,1 \pm 0,2$ & $1,0 \pm 0,1$ & $0,78 \pm 0,11$ \\
\hline 20 & $0,9 \pm 0,2$ & $0,7 \pm 0,2$ & $1,0 \pm 0,1$ & nd \\
\hline 21 & $0,7 \pm 0,1$ & $0,7 \pm 0,2$ & $0,8 \pm 0,1$ & nd \\
\hline 22 & $1,7 \pm 0,2$ & $1,1 \pm 0,1$ & $1,7 \pm 0,1$ & nd \\
\hline 23 & $1,3 \pm 0,1$ & $1,2 \pm 0,1$ & $1,4 \pm 0,2$ & nd \\
\hline 24 & $0,98 \pm 0,04$ & $1,0 \pm 0,3$ & $1,2 \pm 0,2$ & nd \\
\hline 25 & $0,7 \pm 0,1$ & $0,8 \pm 0,1$ & $1,0 \pm 0,1$ & nd \\
\hline
\end{tabular}

*nd = não determinado.

A Figura 1a apresenta os resultados de PCA referentes ao Walkley-Black. Segundo os valores de PC1, a Figura 1b, referente à Análise Elementar, agrega maior quantidade de informação sobre o sistema $(95,10 \%)$. Contudo, através da Figura 1c, referente à análise de TOC, consegue-se visualizar mais claramente as diferenças entre as amostras. A PC1 $(89,95 \%)$ pode ser responsável pela diferenciação entre a origem dos solos, enquanto a PC2 (10,05\%) pode estar relacionada à diferenciação entre os sistemas de manejo e profundidade. A Figura 1d, referente aos métodos de Walkley-Black, Análise Elementar e TOC, mostra que a PC1 $(78,95 \%)$ pode ser responsável pela diferenciação entre os tipos de solos, enquanto a PC2 $(17,13 \%)$ deve estar relacionada com a área de origem. $\mathrm{Na}$ $\mathrm{PC} 1$ as faixas de LVef, 1 a 17, apresentam-se no segundo e terceiro quadrantes, contrastando com as faixas de LVd, 18 a 25 , que se apresentam no primeiro e quarto quadrantes. $\mathrm{Na}$ PC2 as faixas da área experimental de Ribeirão Preto, 1 a 9, apresentaram-se no segundo quadrante, diferenciando das demais faixas da área experimental de Jaboticabal.

Pela Tabela 3, a análise via TOC apresentou resultados mais satisfatórios, com menor coeficiente de variação se comparada ao Walkley-Black e à Análise Elementar. Isto se deveu provavelmente à maior massa de amostra analisada via TOC se comparada à aná-
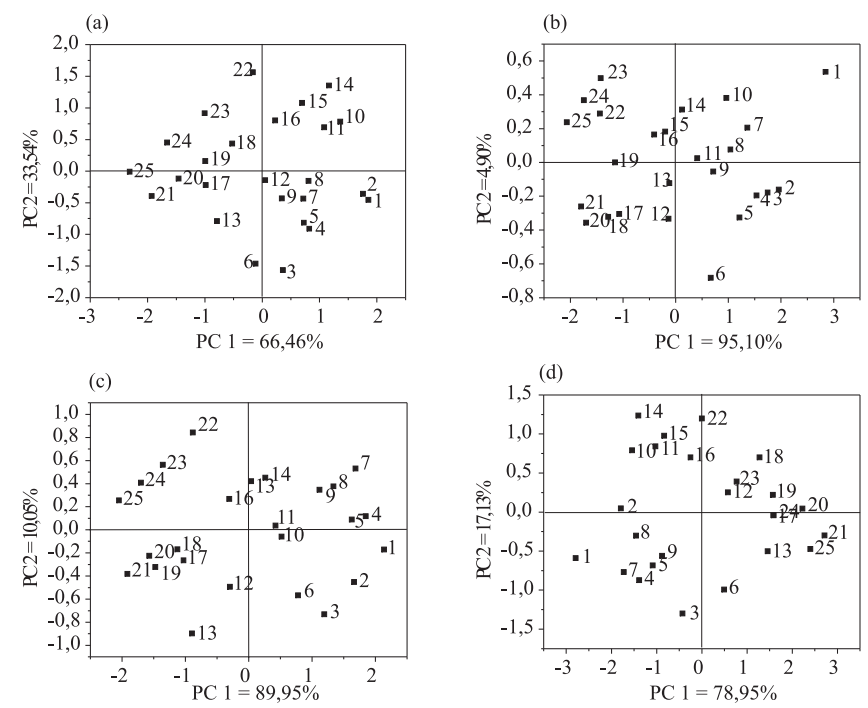

Figura 1. Análise de componentes principais: PC1 x PC2 Scores das metodologias de determinação de carbono por (a) Walkley-Black, (b) Análise elementar, (c) TOC e (d) Walkley-Black, análise elementar e carbono orgânico total (TOC) para determinação do carbono em amostras de solo

lise elementar (100 e $1 \mathrm{mg}$, respectivamente) e também ao fato da oxidação da MO ser mais eficiente no procedimento de TOC, quando comparado com o método de Walkley-Black.

Tabela 3. Coeficiente de variação dos resultados obtidos para determinação de carbono pelos métodos de Walkley-Black, análise elementar, carbono orgânico total (TOC) e análise termogravimétrica (TGA)

\begin{tabular}{|c|c|c|c|c|}
\hline \multirow[t]{2}{*}{ Amostras } & \multicolumn{4}{|c|}{ Coeficiente de Variação (CV) } \\
\hline & $\begin{array}{c}\text { Walkley- } \\
\text { Black }\end{array}$ & $\begin{array}{l}\text { Análise } \\
\text { Elementar }\end{array}$ & TOC & TGA \\
\hline 1 & 15,7 & 17,6 & 12,0 & 3,3 \\
\hline 2 & 10,1 & 8,2 & 13,3 & 0,8 \\
\hline 3 & 14,3 & 21,5 & 21,4 & nd \\
\hline 4 & 31,1 & 12,3 & 5,9 & 0,4 \\
\hline 5 & 14,2 & 12,1 & 0,4 & nd \\
\hline 6 & 18,1 & 16,8 & 20,9 & nd \\
\hline 7 & 3,5 & 5,8 & 4,2 & 2,8 \\
\hline 8 & 19,6 & 5,7 & 13,1 & nd \\
\hline 9 & 12,0 & 10,9 & 4,8 & nd \\
\hline 10 & 21,1 & 21,1 & 15,8 & nd \\
\hline 11 & 16,7 & 16,7 & 5,3 & nd \\
\hline 12 & 23,1 & 23,1 & 7,1 & nd \\
\hline 13 & 11,1 & 11,1 & 10,0 & nd \\
\hline 14 & 15,0 & 15,0 & 10,0 & nd \\
\hline 15 & 22,2 & 22,2 & 10,5 & nd \\
\hline 16 & 12,5 & 12,5 & 11,8 & 0,9 \\
\hline 17 & 10,0 & 10,0 & 8,3 & 3,4 \\
\hline 18 & 15,4 & 15,4 & 8,3 & 12,2 \\
\hline 19 & 18,2 & 18,2 & 10,0 & 14,1 \\
\hline 20 & 22,2 & 22,2 & 10,0 & nd \\
\hline 21 & 14,3 & 14,3 & 12,5 & nd \\
\hline 22 & 11,8 & 11,8 & 5,9 & nd \\
\hline 23 & 7,7 & 7,7 & 14,3 & nd \\
\hline 24 & 4,1 & 4,1 & 16,7 & nd \\
\hline 25 & 14,3 & 14,3 & 9,1 & nd \\
\hline CV médio & 15,1 & 14,0 & 10,5 & 4,7 \\
\hline
\end{tabular}

*nd = não determinado. 
A Figura 2 apresenta um diagrama termogravimétrico característico de uma amostra de solo. Nele observam-se 4 regiões de perda de massa no intervalo de 25 a $600{ }^{\circ} \mathrm{C}$. A primeira região mostra a perda de massa referente à água livre ou água de volatilização. Na segunda, a perda de massa é devida à decomposição da MO, principalmente dos grupos funcionais carboxílicos e fenólicos dos ácidos húmicos, fúlvicos, hidrocarbonetos e MO volátil. Neste intervalo, a perda de massa também pode ser devida à liberação de compostos orgânicos de baixo peso molecular. A terceira região apresenta a perda de massa principalmente devida à oxidação de carbono da $\mathrm{MeO}\left(\mathrm{R}-\mathrm{CH}+\mathrm{O}_{2} \rightarrow\right.$ $\left.\mathrm{CO}_{2}+\mathrm{H}_{2} \mathrm{O}\right)$. Finalmente, na quarta, a perda de massa é devida à perda de água de constituição de hidróxidos metálicos $(\mathrm{Me}-\mathrm{OH} \rightarrow \mathrm{MeO}+$ $\mathrm{H}_{2} \mathrm{O}$, onde $\mathrm{Me}=$ metais como $\mathrm{Al}, \mathrm{Fe}, \mathrm{Mn}$ e outros) $)^{2,8}$.

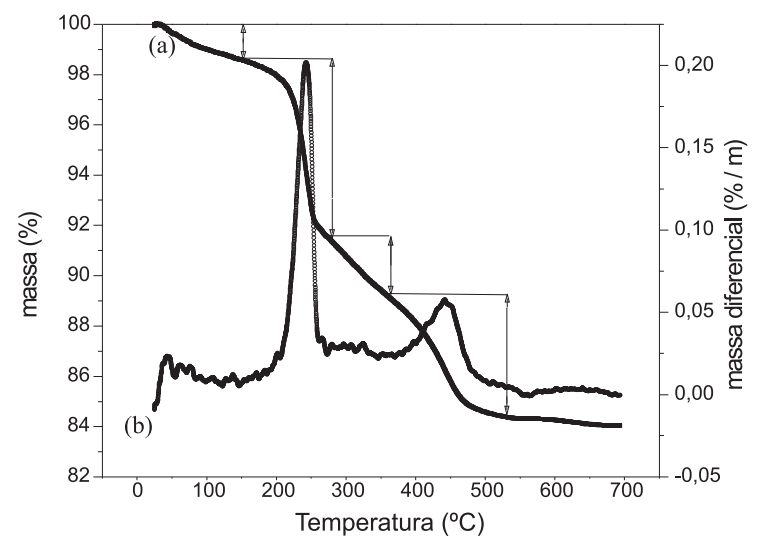

Figura 2. Diagrama termogravimétrico das 4 regiões características no intervalo de 25 a $600{ }^{\circ} \mathrm{C}$ : primeira região $\left(<110^{\circ} \mathrm{C}\right)$; segunda região $(150$ $\left.280{ }^{\circ} \mathrm{C}\right)$; terceira região $\left(270-370^{\circ} \mathrm{C}\right)$ e quarta região $\left(380-530^{\circ} \mathrm{C}\right)$, para a amostra de solo mantido sob sistema de plantio direto $(P D)$, profundidade 0 $10 \mathrm{~cm}$, de um Latossolo Vermelho eutroférrico (LVef). (a) perda de massa relativa e (b) perda de massa diferencial

Na Figura 3, a PCA mostrou que, com apenas duas componentes principais, é possível descrever $92,65 \%$ dos resultados obtidos, sendo $78,35 \%$ da variância total descrita pela primeira componente principal (PC1) e 14,30\% pela segunda componente principal (PC2). Confirma-se maior proximidade entre os métodos de Análise Elementar e TOC, diferenciando dos demais (Walkley-Black e TGA).

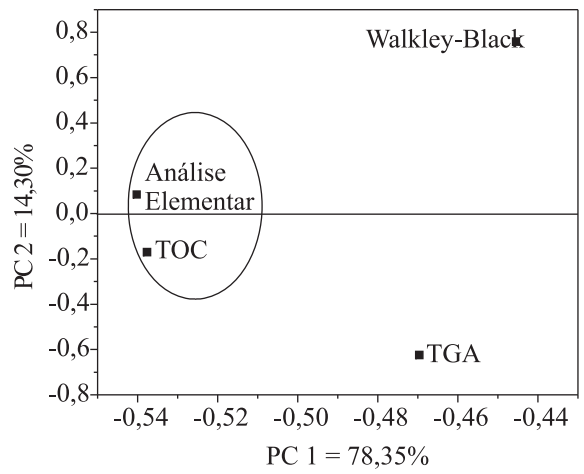

Figura 3. Análise de componentes principais: PC1 x PC2 Loadings correspondentes aos métodos de Walkley-Black, análise elementar, carbono orgânico total (TOC) e análise termogravimétrica (TGA) para determinação de carbono em amostras de solo

\section{CONCLUSÕES}

Dentre os métodos de determinação de carbono, Walkley-Black, TOC e Análise Elementar, o TOC apresentou desvios menores. Isto se deveu provavelmente à maior massa de amostra analisada via TOC se comparada à análise elementar. A oxidação parcial da MO pelo método de Walkley-Black fez com que os valores obtidos fossem em média 22,9 e $17,7 \%$ menores que aqueles observados via TOC e Análise Elementar, respectivamente. Uma visão ambiental mais crítica sugere também a substituição desse método de oxidação por via úmida, visto sua desvantagem relacionada à produção do resíduo que contém crômio em meio fortemente ácido. A dificuldade de distinção entre a perda de água de constituição das argilas e a degradação da MO fez com que os resultados obtidos por termogravimetria apresentassem valores bastantes dispersos, em função das características de cada solo. Os resultados obtidos não inviabilizam o uso de qualquer método de determinação, mas evidenciam a necessidade de uma visão crítica sobre as limitações e vantagens de cada método na determinação de carbono em solos.

\section{AGRADECIMENTOS}

À Embrapa Instrumentação Agropecuária pela infra-estrutura cedida. À Embrapa Pecuária Sudeste, ao Laboratório de Química Ambiental do Instituto de Química de São Carlos, ao Centro de Análises Químicas do Instituto de Química de São Carlos e ao Departamento de Química da Universidade Federal de São Carlos pelas análises dos solos. À FAPESP e ao CNPq pelo auxílio concedido.

\section{REFERÊNCIAS}

1. Reeves, D. W.; Soil and Tillage Research 1997, 43, 131.

2. Miyazawa, M.; Pavan, M. A.; Oliveira, E. L.; Ionashiro, M.; Silva, A. K.; Braz. Arch. Biol. Technol. 2000, 43, 475.

3. Conceição, M.; Manzatto, C. V.; Araújo, W. S.; Martin-Neto, L.; Saab, S. C.; Cunha, T. J. F.; Freixo, A. A.; Comunicado Técnico, Rio de Janeiro: Embrapa Solos 1999, 2, 1.

4. Milori, D. M. B. P.; Galeti, H. V. A.; Martin-Neto, L.; Diekow, J.; GonzálezPérez, M.; Bayer, C.; Salton, J.; Soil Sci. Soc. Am. J. 2006, 70, 57.

5. Walkley, A.; Black, I. A.; Soil Sci. 1934, 37, 29.

6. Jackson, M. L.; Analisis quimico de suelos, Barcelona: Omega, 1982.

7. Jimenez, R. R.; Ladha, J. K.; Commun. Soil Sci. Plant Anal. 1993, 24, 1897.

8. Beyer, L.; Deslis, K.; Vogt, B.; Commun. Soil Sci. Plant Anal. 1998, 29, 1277.

9. http://en.wikipedia.org/wiki/2005, acessada em Novembro 2005.

10. Embrapa - Centro Nacional de Pesquisa de Solos; Sistema Brasileiro de Classificação de Solos, Embrapa Serviço de Produção: Brasília, 1999.

11. Ferreira, E. C.; Rodrigues, S. H. B. G.; Ferreira, M. M. C.; Nóbrega, J. A; Nogueira, A. R.; Eclet. Quim. 2002, 27, 77.

12. Cantarella, H.; Quaggio, J. A; Van Raij, B. Em Análise química para avaliação da fertilidade de solos tropicais; Van Raij, B.; Andrade, J. C.; Cantarella, H.; Quaggio, J. A., eds.; Unicamp: Campinas, 2001, cap. 9. 